\title{
puente sobre el mío QUEQUEN SALADO
}

\section{J. LUISONI y A. A. GIACOBBE, ingenieros}

\section{Argentina}

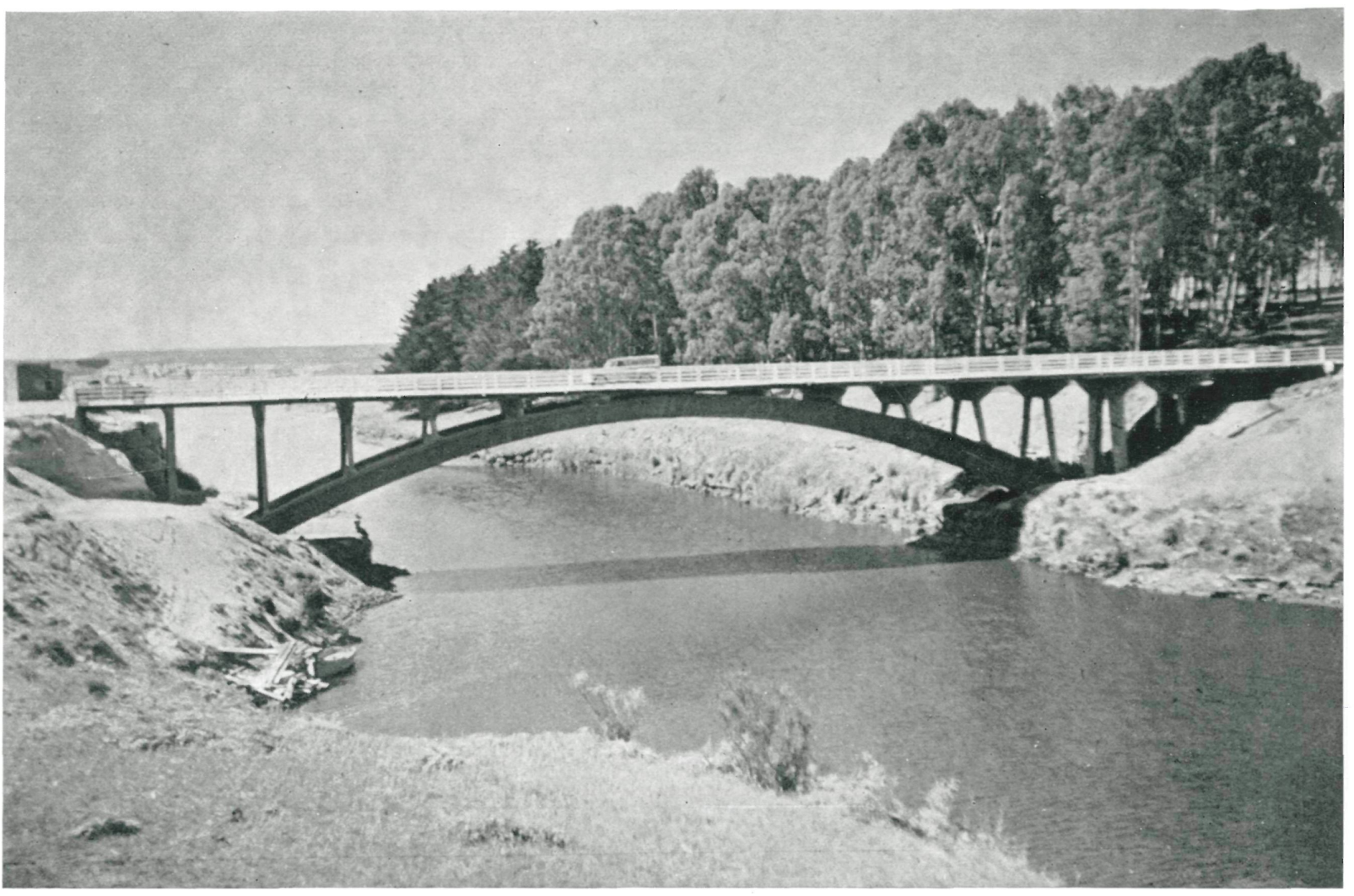

El puente sobre el río Quequén Salado, de hormigón armado, tiene $60 \mathrm{~m}$ de luz en su tramo único y $9 \mathrm{~m}$ de flecha máxima. Los autores del proyecto, que estudiaron con anterioridad la influencia que la forma de una estructura puede tener en la estabilidad y resistencia de la obra, aprovecharon la oporturios de anden teórico que consiste en un solo arco, de hormigón armado, estos én trantesulón da forma quebrada, que por un ado , de has quebrada, que por un lado gana inciamás peconón gran esbel é. diafragmas convenientemente espaciados. Otra particularidad de esta obra la constituye la presencia de dos articulaciones, una para arranque, lo que introduce un estado hiperestático en estas partes de la obra.

\section{Introducción}

Sobre el río Quequén Salado, situado al sureste de la provincia de Buenos Aires (Argentina), se acaba de construir un puente, en arco, laminar y de hormigón armado.

La zona del río en que se ha ubicado el puente, se presenta con una canalización regular y de márgenes rectilíneas.

Para el estudio estructural de esta obra se prestó especial interés a la necesidad de evitar pilares intermedios, ya que dificultan el desagüe normal, pero adoptando formas arquitectónicas que se ajustasen lo mejor posible al paisaje circundante. 


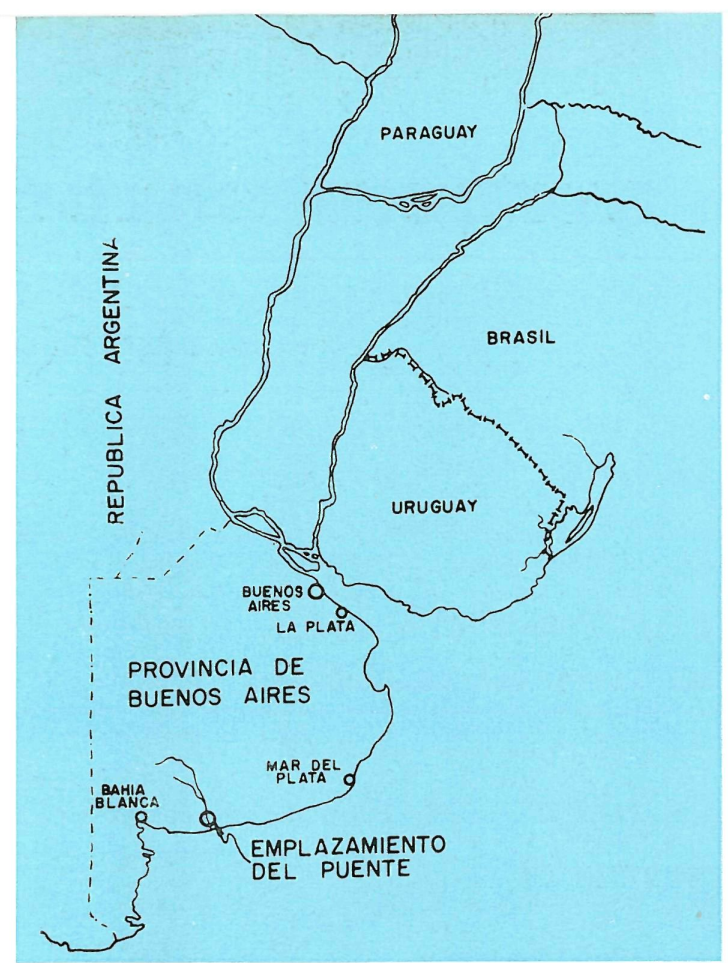

pland de sifunción

Como se dijo anteriormente, se trataba de lograr un gran perfil mojado y altura suficiente para el desagüe. La solución que mayores ventajas presentaba, dada la anchura media del río, era la de un puente en arco, de pronunciada altura sobre aguas y de $60 \mathrm{~m}$ de luz. Admitiendo $9 \mathrm{~m}$ de flecha, la relación flecha-luz resultaba ser de 1/6,66. El arco así concebido debería tener dos articulaciones: una en cada arranque.

Los autores del proyecto trataron de obtener el mayor provecho posible de la forma óptima para este tipo de estructura.

\section{La Bstrutetura}

Es indudable que las formas laminares proporcionan a las estructuras una gran esbeltez y, además, presentan una ventaja respecto a otras cons-

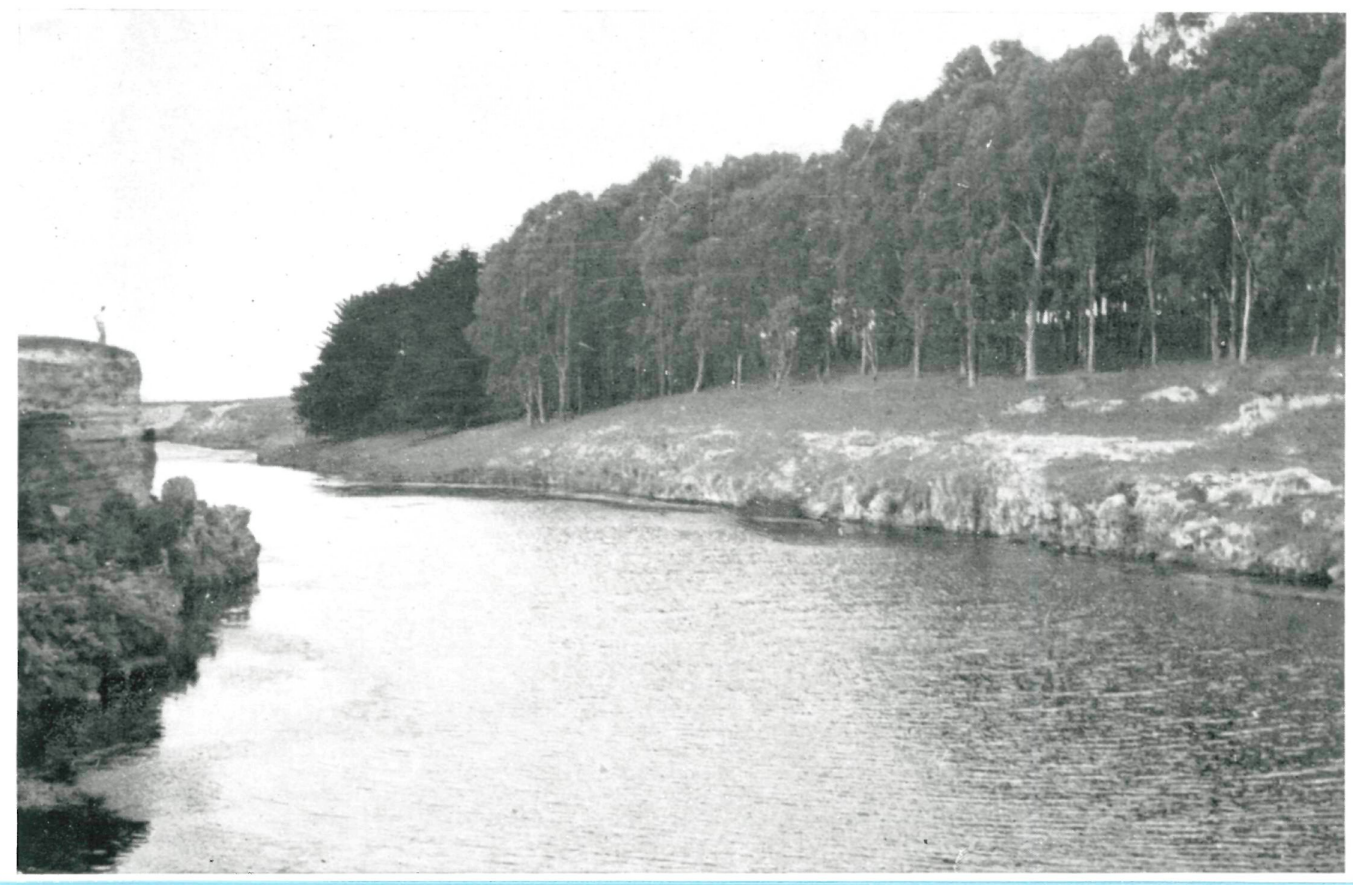

Zona de
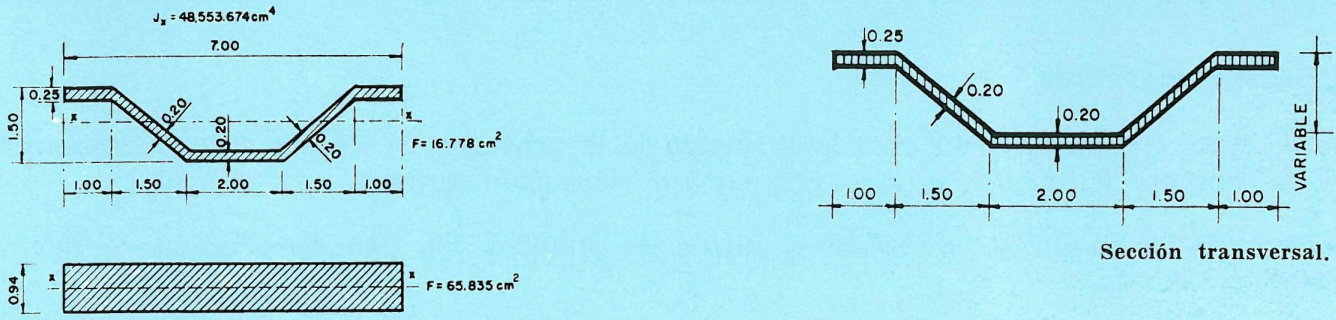

Sección transversal.

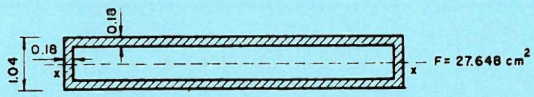

Comparación de secciones de igual momento de inercia. 
Excavación con bombeo. En segundo plano se ve el martinete utilizado para hincar los pilotes de la cimbra.

tituidas por vigas cajón, ya que éstas necesitan de un encofrado interior y una cimbra más pesada. Después de detenidos estudios y análisis de la forma que debía adaptarse, se llegó a la conclusión de que la más indicada sería la de una lámina de sección transversal en forma de línea quebrada y de concavidad. expuesta hacia la parte superior.

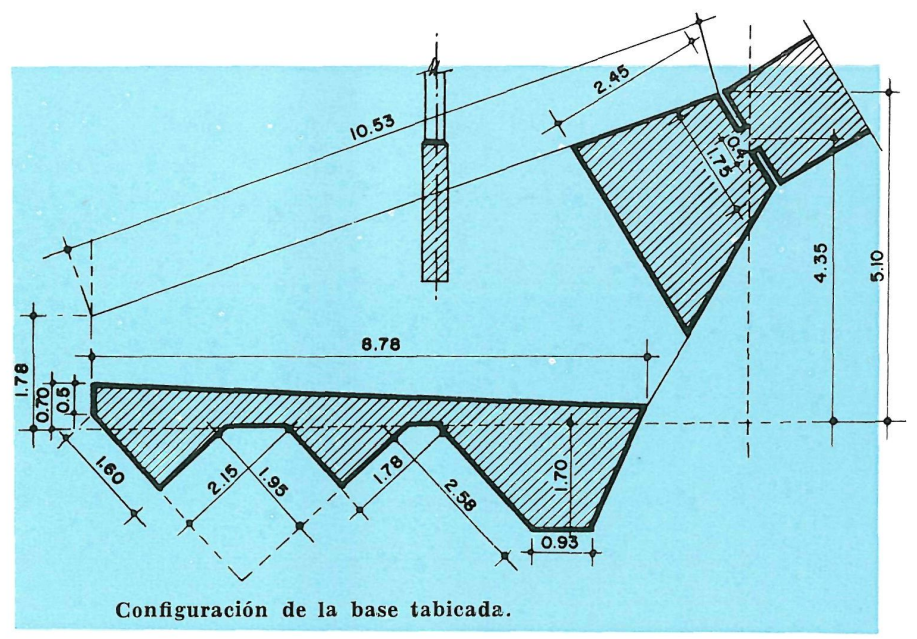

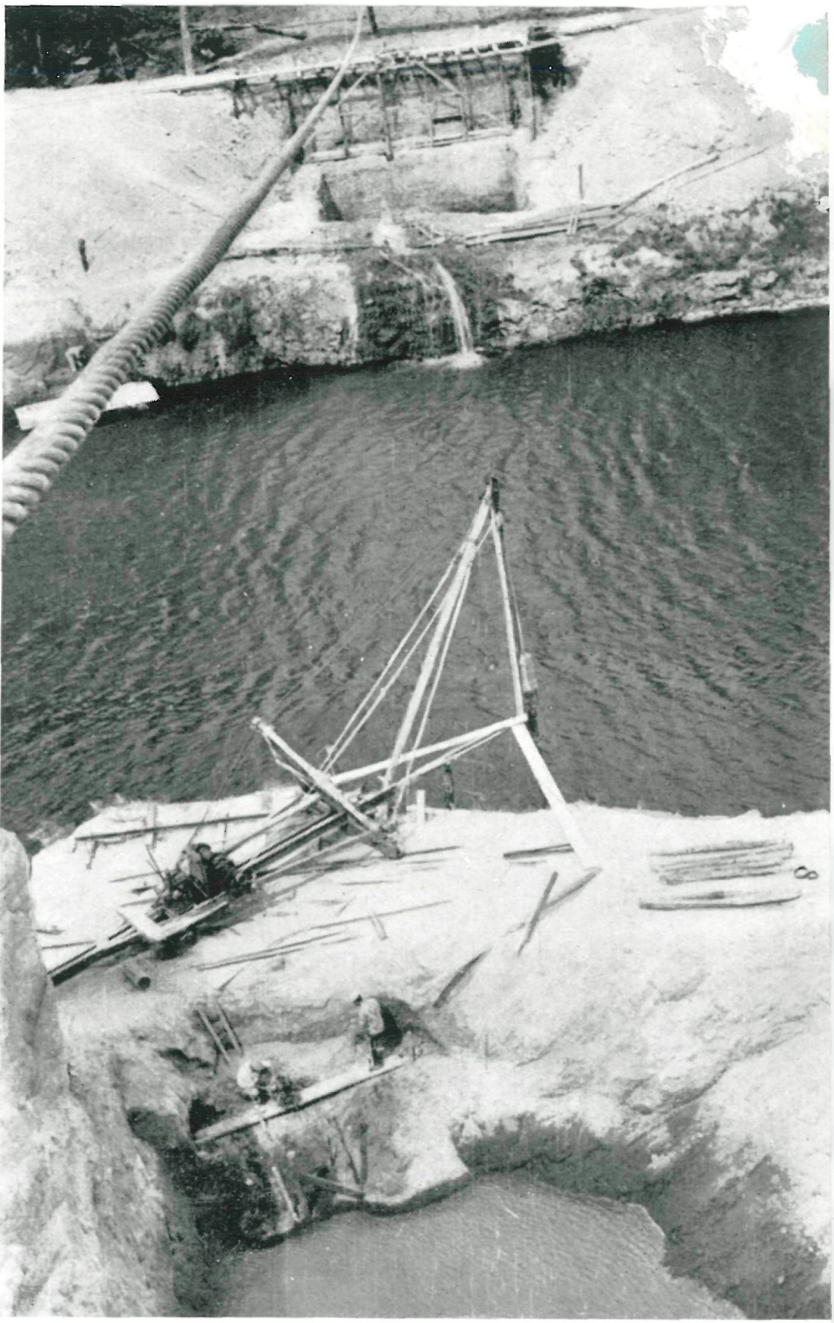

Antes de llegar a esta determinación, se estudiaron comparativamente tres tipos distintos de arco, cuyas secciones transversales correspondieron: a la línea quebrada antes indicada, a una lámina de sección rectangular y a un arco de sección rectangular, hueca y de paredes delgadas.

Este tipo de arco cuenta con una serie de diafragmas espaciados a $5 \mathrm{~m}$, que le dan gran rigidez. Como el tablero se apoya sobre una palizada que arranca del arco, se aprovechó la presencia de estos diafragmas para sobre ellos apoyar la palizada. Estos soportes, que en un principio se previeron constituyendo una sola pared. continua, se sustituyeron por pórticos que le dan menor peso y suficiente rigidez. Como la parte central del

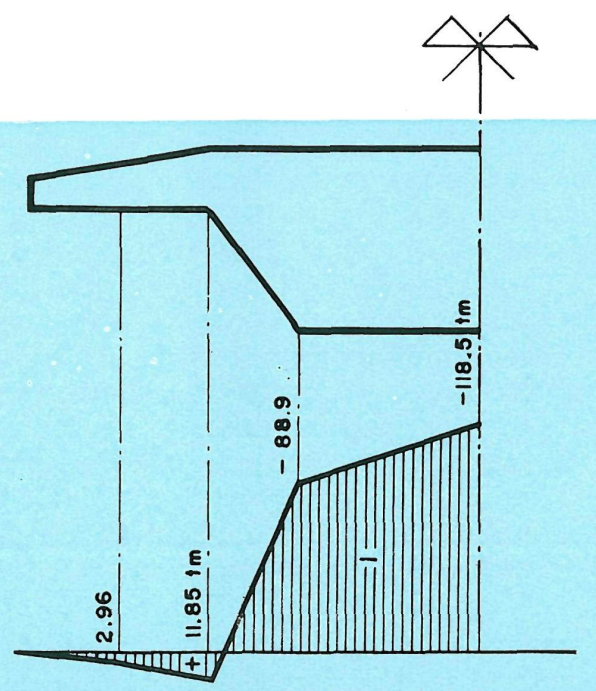

Diagrama de momentos flectores.

Diagrama de carga producido por la armadura longitudinal en tracción.

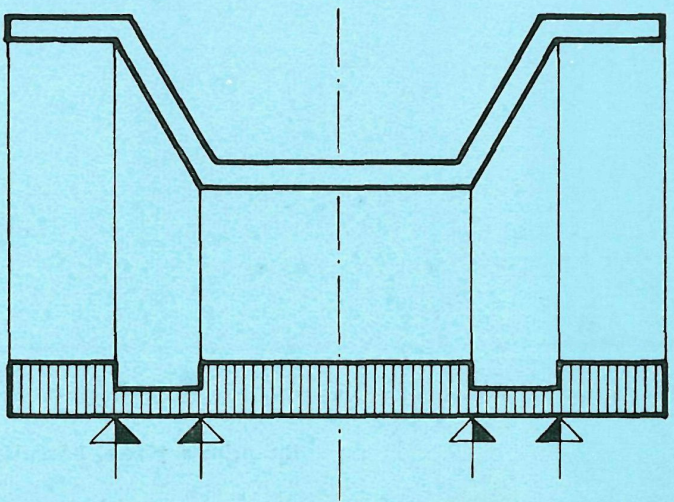




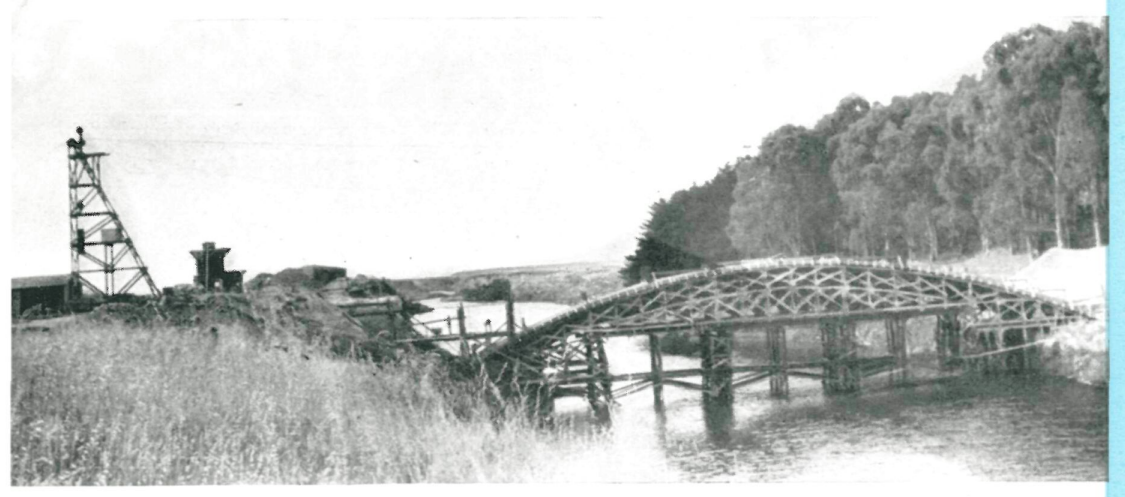

Vista de la cimbra del arco.

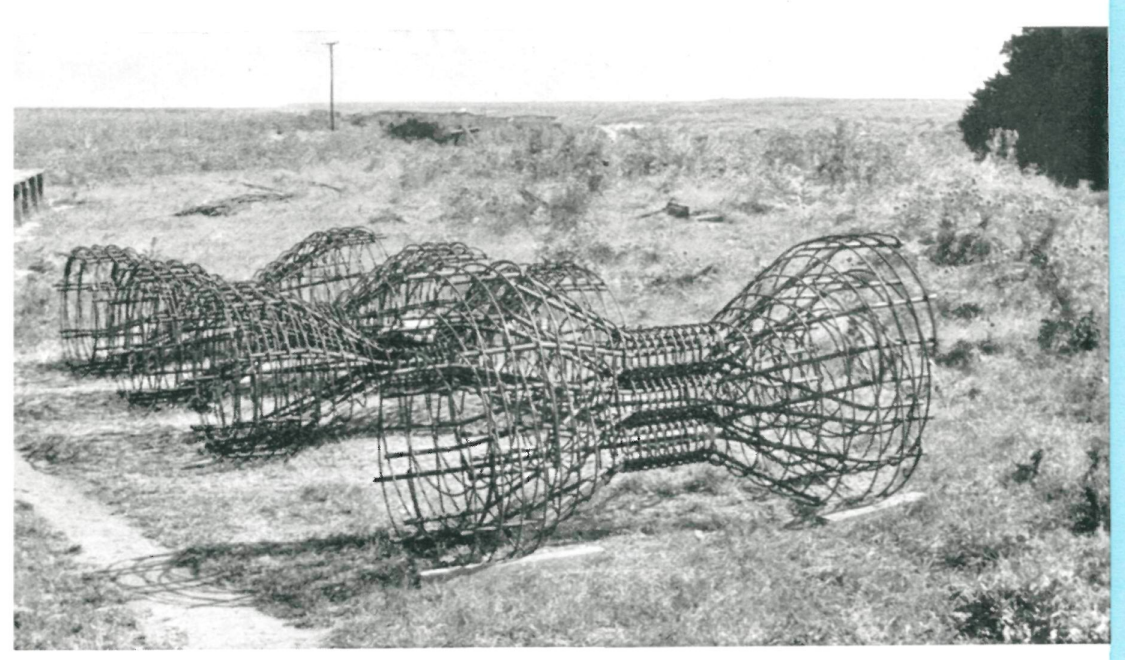

Articulaciones del arco.

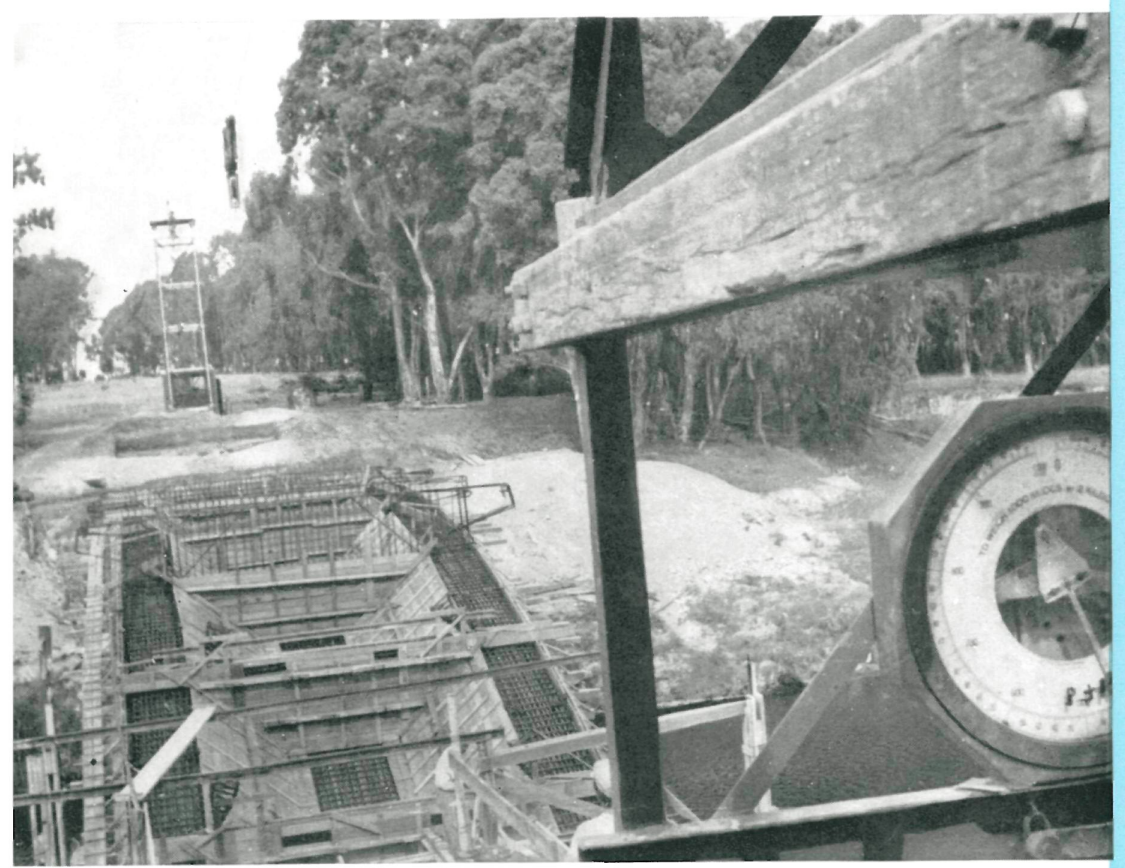

En primer plano, báscula para pesar los materiales.
Corrimientos verticales medidos en el arco.
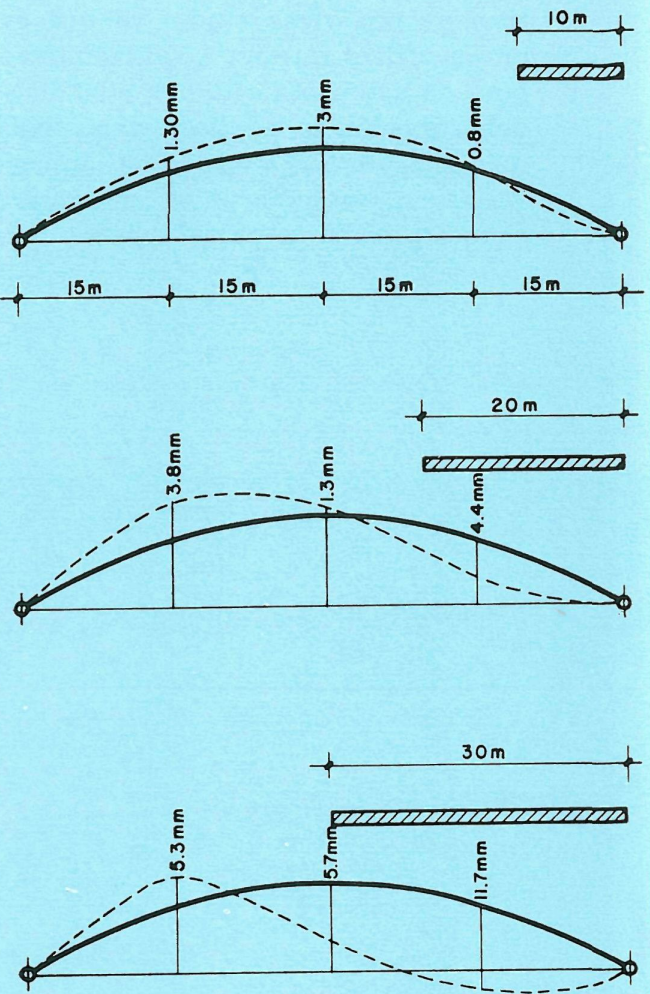

arco presentaba escasa separación con el tablero, los cinco diafragmas correspondientes a esta zona se prolongaron en altura, formando pared continua hasta enrasar con el intradós del tablero.

La losa del tablero tiene $22 \mathrm{~cm}$ de espesor y en su parte inferior se dejó una ligera contraflecha.

A uno y otro lado de la calzada se extienden los andenes, de $1,20 \mathrm{~m}$ de anchura, a excepción de los cinco metros centrales, en los que dicha anchura es de $2,20 \mathrm{~m}$, para dar facilidades a los pescadores que aprovechan el puente para este deporte. El arco, doblemente articulado, tiene un canto creciente de clave a arranques $(1,5 \mathrm{y}$ $1,75 \mathrm{~m}$, respectivamente). La ley de variación de canto es inversamente proporcional al coseno del ángulo que forma la tangente a la directriz del arco con la horizontal. 
Las dos láminas extremas, superiores, de la sección quebrada del arco, tienen $25 \mathrm{~cm}$ de espesor, mientras que el de las inferiores, en toda la longitud, es de $20 \mathrm{~cm}$. El arco en proyección horizontal tiene una anchura de $7 \mathrm{~m}$, y la de la calzada es de 8,30.

En los arranques, y con objeto de lograr espacio suficiente para las articulaciones, se le dio sección rectangular al arco en una zona de transición, de 1,75 $\mathrm{m}$ de longitud, en cuya extremidad aparece la lámina de sección quebrada.

En cada arranque se han colocado dos articulaciones que transmiten un esfuerzo máximo de 975 toneladas. La articulación propiamente dicha está formada con un triple núcleo zunchado.

Los diafragmas, sobre los que se levanta la palizada, tienen asignada una triple función resistente: en primer lugar, dan rigidez al arco y aseguran la constancia de la forma del mismo; en segundo lugar, se utilizan como elementos de repartición de cargas concentradas en los pies de los montantes de la palizada, y, por último, están llamados a absorber los empujes provocados por los referidos montantes.

\section{Cimientos}

Del estudio y reconocimiento del suelo en el lugar de ubicación de la obra se pudo comprobar: que la estructura debía apoyarse sobre un terreno compacto con capacidad de carga de $3,5 \mathrm{~kg} / \mathrm{cm}^{2}$. La profundidad de este banco compacto varía de una a otra margen. Con objeto de conocer las posibilidades que ofrecía el terreno, se procedió a una serie de sondeos de reconocimiento, mediante los cuales se supo que uno de los cimientos se hallaría a distinta cota que el otro.

El cimiento que corresponde a la margen de Copetonas tiene forma convencional, masiva, mientras que el correspondiente a la margen opuesta se halla a $2,65 \mathrm{~m}$ de cota por debajo del anterior, lo que implica un apoyo más alto, ya que los arranques se hallan situados a un mismo nivel. El apoyo más bajo está constituido por una losa de solera, sobre la que se levantaron varios contrafuertes que terminaban en el bloque de las articulaciones.

E1 puente lleva tres tramos de acceso en la margen de Copetonas, mientras que la opuesta sólo tiene uno. Los soportes de estos tramos se apoyan en unas zapatas que descansan directamente sobre el terreno.

\section{Căllcullo del antrọ}

La directriz del arco es una parábola de segundo grado, cuya expresión analítica es:

$$
Y=4 f\left(\varepsilon-\varepsilon^{2}\right),
$$

donde:

$$
\varepsilon=\frac{x}{l}
$$

Admitiendo un empuje determinado y contando la indeterminación hiperestática, se siguió el procedimiento de líneas de influencia para el análisis de las cargas sobre el tablero y esfuerzos que se transmiten al arco en forma concentrada. Para la determinación gráfica de las líneas de influencia se tuvo en cuenta el peso propio y sobrecargas, siendo objeto de consideración independiente el efecto de la variación de temperatura, retracción y corrimiento de apoyo. Para los efectos de la temperatura se admitió un campo de variación de $\pm 40^{\circ} \mathrm{C}$, y para los corrimientos, un incremento de 2 centímetros. 

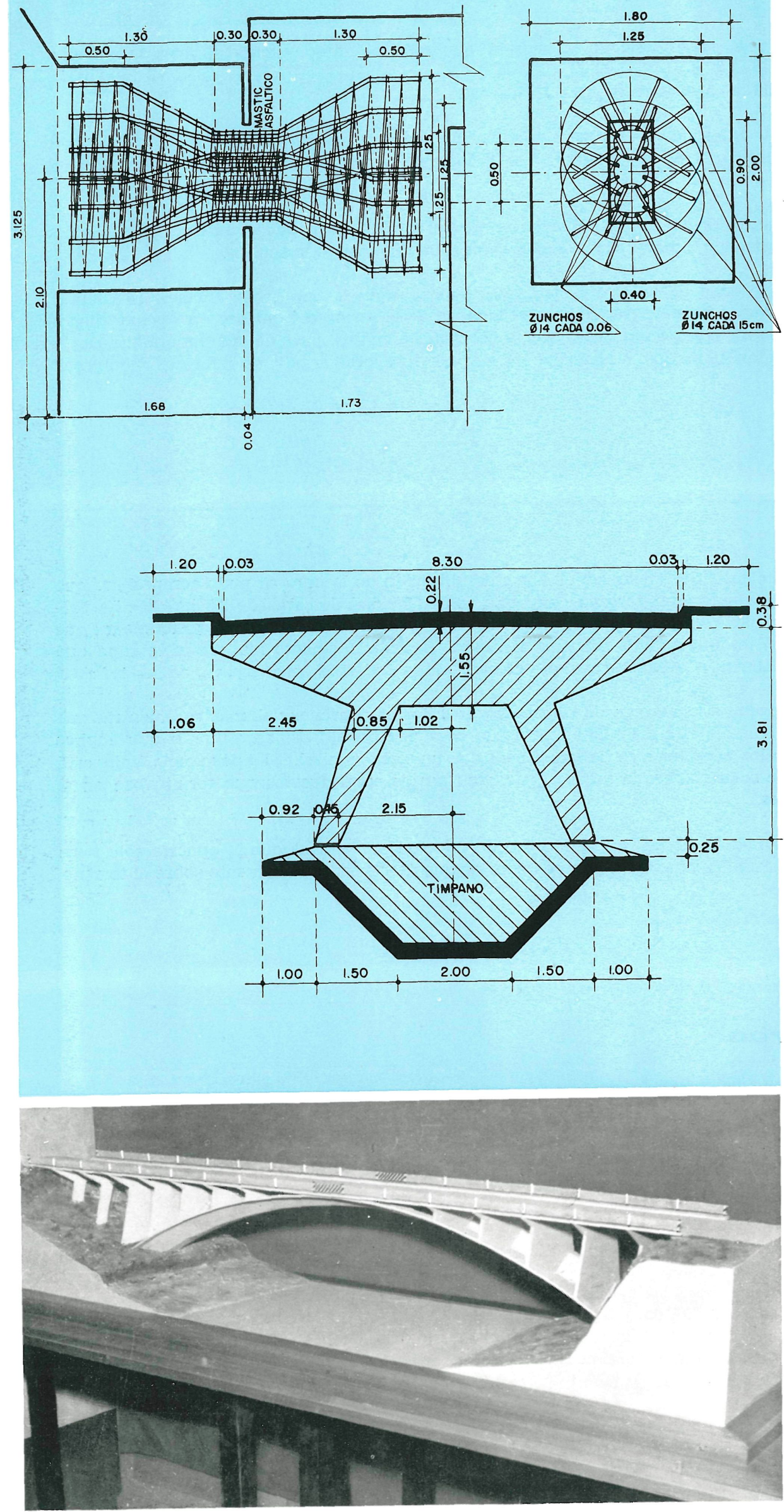

Fueron también objeto de estudio particular los esfuerzos cortantes, acciones transversales, flexión compuesta y lateral, torsión debida al efecto del viento, pandeo y flexión en los montantes, así como toda serie de efectos combinados tratados en las condiciones más desfavorables. El pandeo del arco se verificó tomando un trozo de $5 \mathrm{~m}$ de longitud, que corresponde al espaciamiento de los diafragmas. La seguridad obtenida respecto a la carga crítica es igual a 4, mientras que dicha seguridad, calculada para las láminas superiores, corresponde a 50 .

\section{Ejecunción}

La obra se adjudicó a la empresa CODISA, de la ciudad de La Plata, y la dirección corrió a cargo del ingeniero Francisco Mosetiz. Las excavaciones para cimientos se realizaron teniendo que emplear permanentemente un equipo de bombeo para el agotamiento, ya que las infiltraciones del río y de la capa freática aforaban un gasto de consideración que hubiera anegado rápidamente las zonas excavadas.

Como la afluencia de agua, por los motivos antes indicados, era continua y de consideración, las excavaciones de cimientos se realiza-

Vista y corte de una articulación.

Pórtico biarticulado

y tímpano.

Maqueta

del anteproyecto. 
Sección transversal de la calzada.

Arco de altura variable adoptado.

El puente, en servicio.

Vista próxima del extradós del arco.

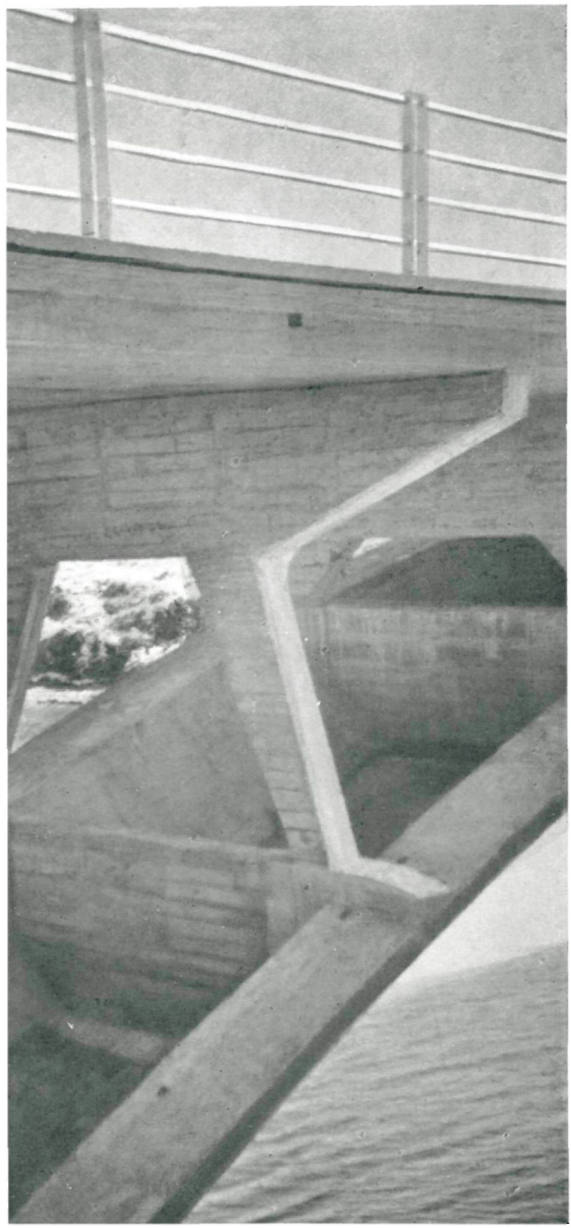

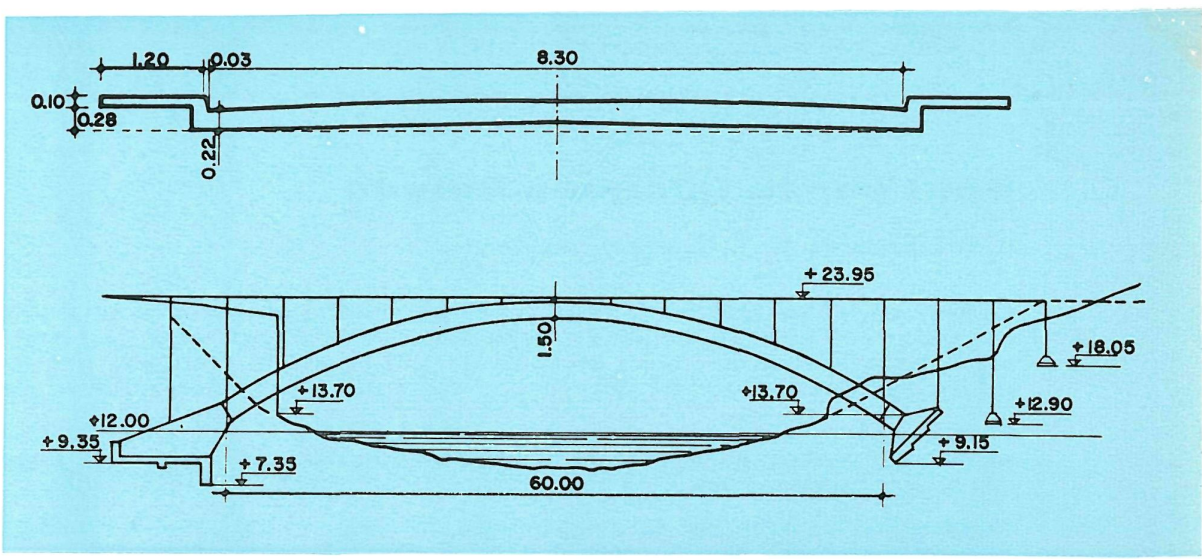

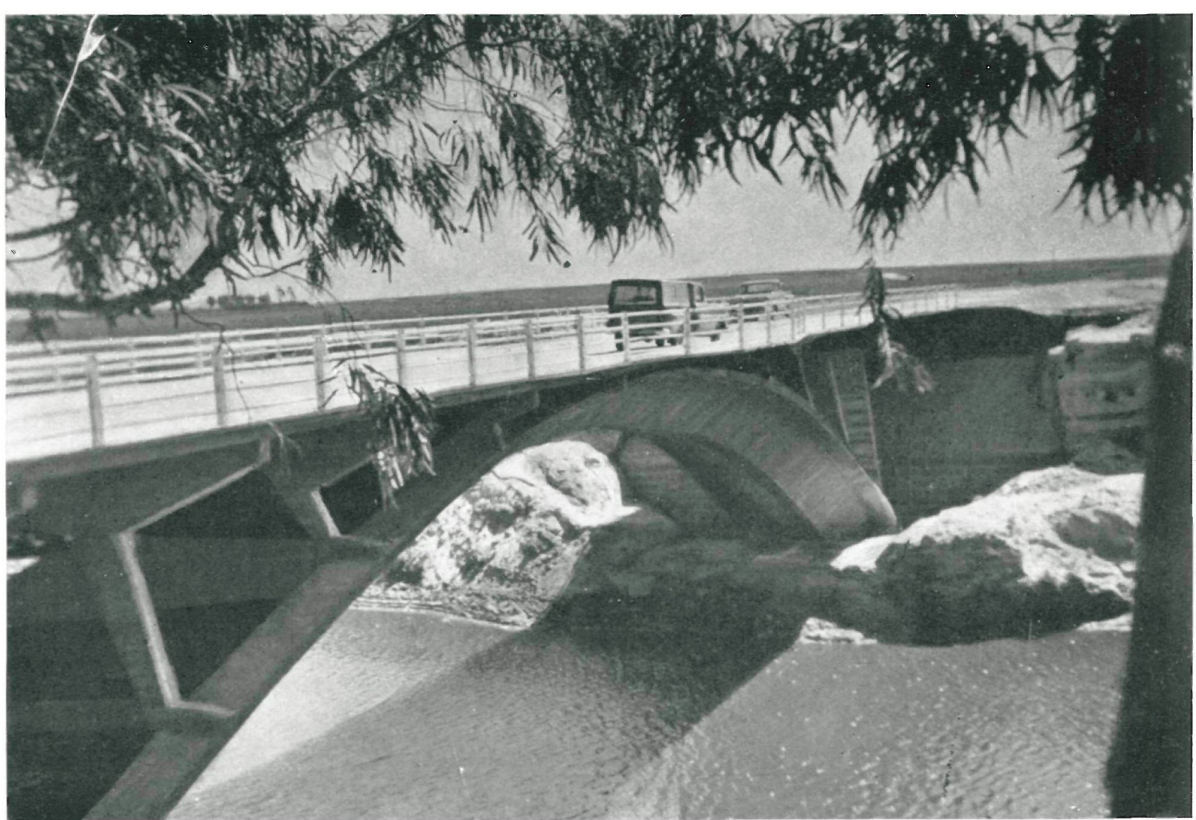

ron abriendo un calderín-guía que iba recogiendo las aguas de infiltración a medida que se descendía en profundidad a la cota prevista para el apoyo de cimientos. El descenso hasta llegar a la referida cota se protegió con una serie de tablestacas de madera que aseguraban la estabilidad de los hastiales hasta el momento de hormigonar.

Para facilitar el transporte de materiales se tenđió sobre el lugar de ubicación de obras una especie de cable carril o blondín, el cual se utilizó para el transporte de la madera del encofrado, armaduras, hormigón y para el desencofrado.

La cimbra se apoyó sobre una serie de pilotes hincados en el fondo del cauce del río. Tanto los pilotes como los demás elementos constitutivos de la cimbra eran de madera y, en particular, de eucalipto para los pilotes. 


\section{Pomt Sur Ix Quequen Salado}

C. J. Luisoni et A. A. Giacobbe, ingénieurs.

Le pont, en béton armé, sur la Quequen Salado, a $60 \mathrm{~m}$ de portée en un seul tronçon, et $9 \mathrm{~m}$ de flèche maximale. Les auteurs du projet qui étudièrent antérieurement l'influence que la forme d'une structure pourrait exercer sur la stabilité et la résistance de l'ouvrage, ont profité de l'occasion qui se présentait à eux pour constater expérimentalement les idées entrevues au cours des études, d'ordre théorique, effectuées.

Tous ces essais ont eu pour résultat la solution adoptée qui consiste en un seul arc, de béton armé, de section transversale polygonale ou discontinue qui, d'un côté, gagne de l'inertie et, d'un autre, se rapproche des sections creuses. Son poids est réduit et son élancement considérable.

Etant donné l'allégement de poids propre, le cintre s'avère plus économique; et la rigidité se voit considérablement augmentée par l'introduction de diaphragmes dûment espacés.

Une autre particularité de cet ouvrage est la présence de deux articulations, une par arrachement, ce qui introduit un état hyperstatique dans ces parties de l'ouvrage.

\section{Bridge over the Quequen Saldado River}

C. J. Luisoni and A. A. Giacobbe engineers.

This bridge has a single span, $60 \mathrm{~m}$ in length, and has a maximum rise of $9 \mathrm{~m}$. It is made of reinforced concrete.

In this project the authors tested in practice a number of theoretical investigations which they had previously carried out on the influence which the shape of a structure has on its strength and stabilty.

They finally arrived at a solution consisting of a single reinforced concrete arch, of polygonal outline, which thereby has an increased moment of inertia, and is more similar to a hollow section. It has less weight, and is very slender.

Due to the reduction in weight, the formwork is cheaper. and the stiffness is considerably improved by the addition of a series of suitably spaced diaphragms.

Another feature of this project is that the arch has two hinges, one at each springer, and this causes part of the structure to be hyperstatic.

\section{Brilike über den Quenquen Sulado Fluss}

G. J. Luisoni und A. A. Giacobbe. Ingenieure.

Die Spannbetonbrücke über den Quénquen Salado hat eine Spannweite von $60 \mathrm{~m}$ und einen maximalen Stich von $9 \mathrm{~m}$.

Die Autoren dieses Projektes, die zunächst den Einfluss der Konstruktionsform auf die Stabilität und den Widerstand des Bauwerkes genau untersuchten, nutzten die Gelegenheit, die sich ihnen bot, die theoretisch ausgearbeiteten Ideen experimentell nachzuweisen.

Aus all diesen Versuchen ist dann die durchgeführte Lösung entstanden, die einen einzigen aus Spannbeton gefertigten Bogen vorsieht, dessen Querschnitt eine poligone gebrochene Form hat. Dadurch gewinnt sie auf der einen Seite an Trägheit, doch auf der anderen Seite gleicht sie sich den hohlen Querschnitten an. Die Brücke ist von nur geringem Gewicht und sehr schlank.

Auf Grund der Reduzierung des Eigengewichtes war das Lehrgerüst wesentlich billiger; die Steifheit der Brücke hat man noch durch entsprechend eingebaute Querschotte erhöht.

Eine weitere Besonderheit dieses Bauwerkes ist das Vorhandensein von Gelenken in den Kämpfern, die die ganze Konstruktion in diesen Teilen statisch unbestimmbar machen. 